\title{
DIAGNOSTIC ACCURACY OF ULTRASOUND AND COMPUTED TOMOGRAPHY IN DETECTION OF PANCREATIC TUMOURS
}

\author{
Somasekhar R1, Praveen Elaprolu²
}

${ }^{1}$ Assistant Professor, Department of Radiodiagnosis, Shri Sathya Sai Medical College and Research Institute, Kancheepuram. 2Postgraduate Student, Department of Radiodiagnosis, Konaseema Institute of Medical Sciences and Research Institute, Amalapuram.

\section{ABSTRACT}

\section{BACKGROUND}

The aim of the study is to assess the accuracy of ultrasound and CT in the evaluation of pancreatic tumours \& in determining the resectability and prognosis of various pancreatic tumours.

\section{MATERIALS AND METHODS}

This study spanned a course of 18 months (January 2015-June 2016), which included 50 cases. Patients with clinical complaints and laboratory results suspicious of pancreatic tumours were evaluated with ultrasound and CT.

\section{RESULTS}

The sensitivity and specificity of contrast enhanced computed tomography in the diagnosis of pancreatic tumours is $88.37 \%$ and $71.43 \%$ respectively. The sensitivity and specificity of ultrasound in the diagnosis of pancreatic tumours is $76.10 \%$ and $62.50 \%$ respectively.

\section{CONCLUSION}

Ultrasound was less sensitive and specific in the detection of pancreatic tumours when compared to contrast enhanced computed tomography.

\section{KEYWORDS}

Pancreatic Tumours, Ultrasound, Contrast Enhanced Computed Tomography.

HOW TO CITE THIS ARTICLE: Somasekhar R, Elaprolu P. Diagnostic accuracy of ultrasound and computed tomography in detection of pancreatic tumours. J. Evolution Med. Dent. Sci. 2017;6(3):251-254, DOI: 10.14260/Jemds/2017/57

\section{BACKGROUND}

CT is the most versatile technique for imaging pancreas. It has a high diagnostic accuracy for determining the nature of neoplasms. CT has an advantage over ultrasound in the evaluation of pancreas. Pancreas being retroperitoneal may be obscured by bowel gas which makes it difficult to visualise on ultrasound. But CT has no such limitations. The entire anatomy of pancreas and pathology can be well delineated by CT.

The anatomical details are best obtained using $5 \mathrm{~mm}$ scan collimation and $3 \mathrm{~mm}$ at specific areas if required. Water is preferred as an oral contrast agent. $500-900 \mathrm{~mL}$ given 30 minutes before and $200-300 \mathrm{~mL}$ given immediately before the scan. ${ }^{1} 150-180 \mathrm{~mL}$ of intravenous contrast is administered as a bolus through a peripheral vein. The bolus is given in a uniphasic $(2.5 \mathrm{~mL} / \mathrm{sec})$ or biphasic manner $(2.5$ $5 \mathrm{~mL} / \mathrm{sec}$ ) for a total of $50 \mathrm{~mL}$ and then $1 \mathrm{~mL} / \mathrm{sec}$ until a total of $150-180 \mathrm{~mL}$ is given. The scan sequence is initiated $30-40$ seconds after the bolus injection has been started. This bolus contrast technique causes the pancreatic parenchymal attenuation to increase by about 50-60 HU over the

Financial or Other, Competing Interest: None.

Submission 21-10-2016, Peer Review 27-12-2016,

Acceptance 02-01-2017, Published 09-01-2017.

Corresponding Author:

Dr. Somasekhar R,

D. No. 19-7-15,

Opp. P. A. Quarters,

Kanukurthi Vari Street,

Vizianagaram-535002

E-mail: somarudrabhatla@gmail.com

DOI: $10.14260 /$ jemds $/ 2017 / 57$ non-contrast baseline, thus aiding the detection of small hypervascular/hypovascular pancreatic masses. In addition, contrast enhancement of the peripancreatic blood vessels facilitates diagnosis, particularly when assessing the resectability of neoplasms.

CT of the pancreas should also include evaluation of the liver and the remaining abdomen. This is important for detecting metastases from primary pancreatic neoplasms and for identifying any incidental pathology or other abnormalities that can mimic pancreatic diseases.

Using high-resolution USG, real-time equipment with frequencies up to $7.5 \mathrm{MHz}$, the major part of the pancreas can be visualised in $95 \%$ of the patients. Sonographic examination of the pancreas should begin with the patient in the supine position. Transverse scan in the midline below the xiphoid is done by moving downwards, towards the feet using the related vascular landmarks to identify the region of the pancreas. The probe is turned oblique to visualise the gland in its entirety.

Using the left kidney as an acoustic window, the tail of pancreas may be visualised anterior to its upper pole in a left coronal plane. The tail of pancreas can also be seen adjacent to the spleen from the left lateral intercostal approach using a coronal plane. The head can be seen from the right lateral approach on a coronal plane.

Increasing stomach distension with fluid provides an acoustic window, causes movement of the intragastric gas and displaces the gas filled colon and small bowel loops inferiorly. The water filled stomach provides an excellent window for visualising pancreatic tail, the most difficult portion of the pancreas to visualise sonographically. 


\section{MATERIALS \& METHODS}

This is a prospective study of 50 patients with pancreatic lesions. In this study, an attempt was made to evaluate the efficiency of ultrasound and CT in detecting and diagnosing pancreatic neoplasms.

The appearance of pancreatic lesions on ultrasound and CT and their correlation with age and sex and different clinical settings were studied. The patients were referred to our department, from the departments of Surgery, Medicine, and Paediatrics with a clinical suspicion of pancreatic pathology.

\section{Source of Data}

The main source of data for this study was patients from the teaching hospital attached to KIMS Medical College, Amalapuram.

This study was conducted at the Department of Radiodiagnosis, KIMS Hospital, Amalapuram during a period of 18 months (January 2015-June 2016), which included 50 cases.

\section{Selection of Patients}

Most of the patients presented with epigastric and right hypochondrial pain, vomiting, loss of weight and loss of appetite.

Patients with clinical complaints and laboratory results suspicious of pancreatic neoplasms, were further evaluated with ultrasound and CT. The ultrasound equipment used was ClearVue 650 of Philips, Logic 400 of GE and Adara of Siemens. The Computed Tomography scanner used was GE High-Speed Spiral/Helical CT.

After USG \& CT scan, the cases were subjected to further imaging with MRI with DWI Sequence, MRCP, Endoscopic US \& FDG PET Scan.

MRI has better contrast resolution than MDCT and is superior in detecting small tumours and metastases. ${ }^{2}$ The use of DW MRI may allow earlier detection of pancreatic tumours and metastases in the liver and lymph nodes. ${ }^{3-4}$

Endoscopic US has a recognised role in the detection and staging of small tumours. It can help detect masses as small as $0.2 \mathrm{~cm}$. Endoscopic US can clarify equivocal findings at MDCT or MRI and allows biopsy of suspect lesions.

PET is an emerging technique for characterising tissue on the basis of functional rather than morphologic information. The principle of FDG PET is that malignant tissues have greater uptake and retention of FDG than does normal tissue due to enhanced glucose metabolism. Pancreatic adenocarcinoma generally shows intense focal FDG uptake. The biggest potential impact of FDG PET is in the detection of small metastases, an area in which MDCT and MRI generally underestimate lesions. ${ }^{5}$

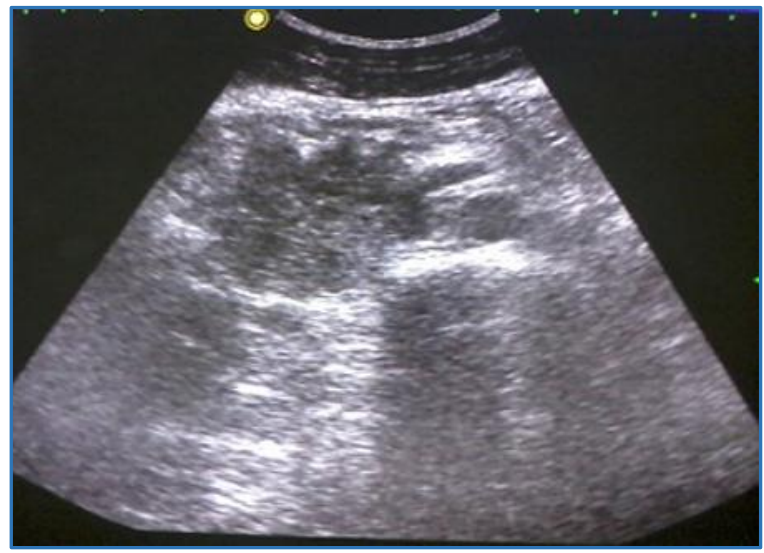

Figure 1a

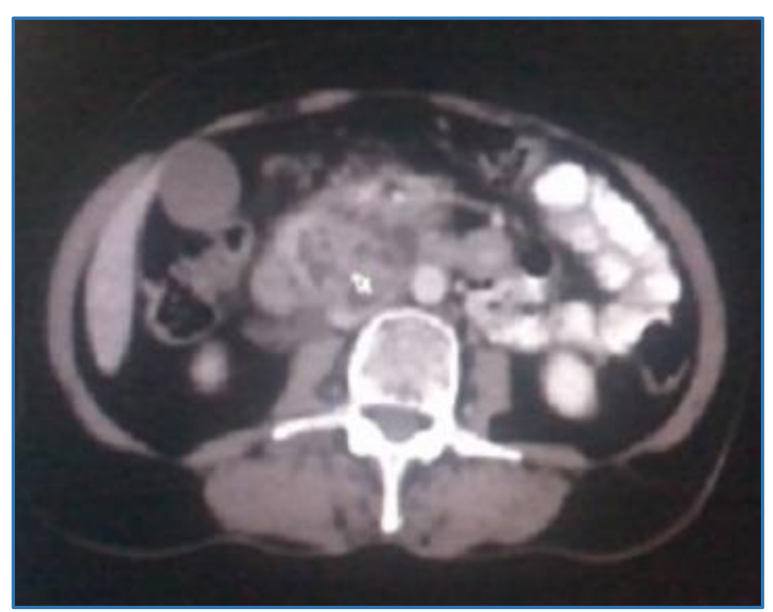

Figure 1a \& b. Mass Lesion in Head of Pancreas with Dilated CBD and Pancreatic Duct

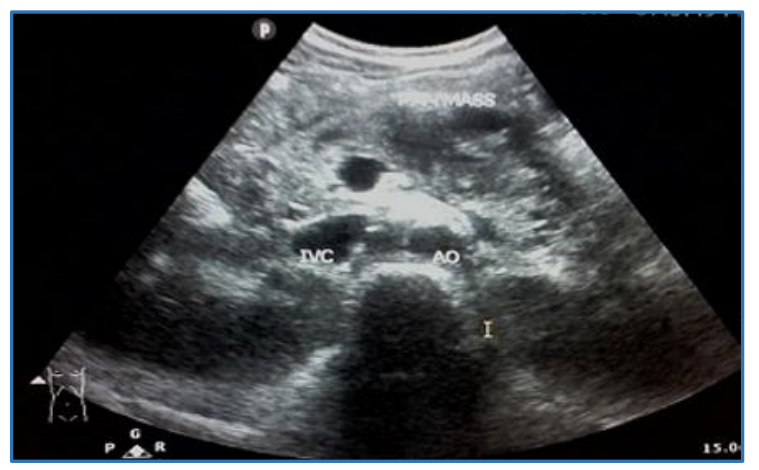

Figure 2a

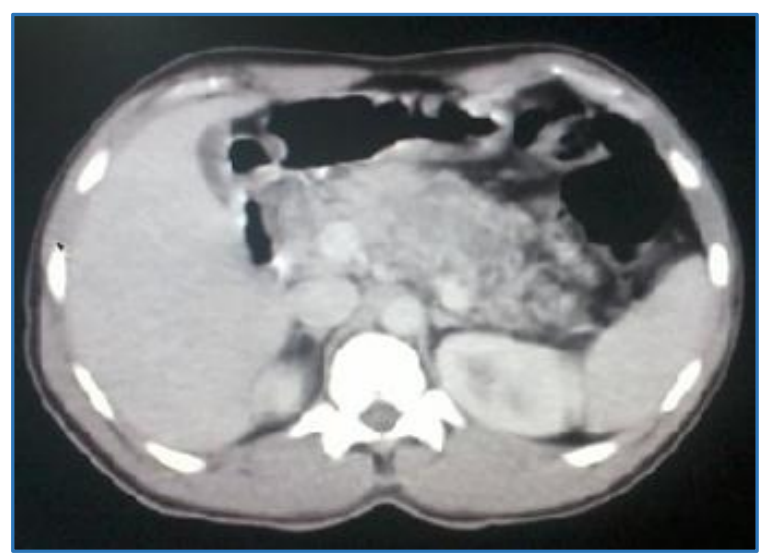

Figure 2a \& b. Ill-defined Mass in the Body of Pancreas with Lymphadenopathy 


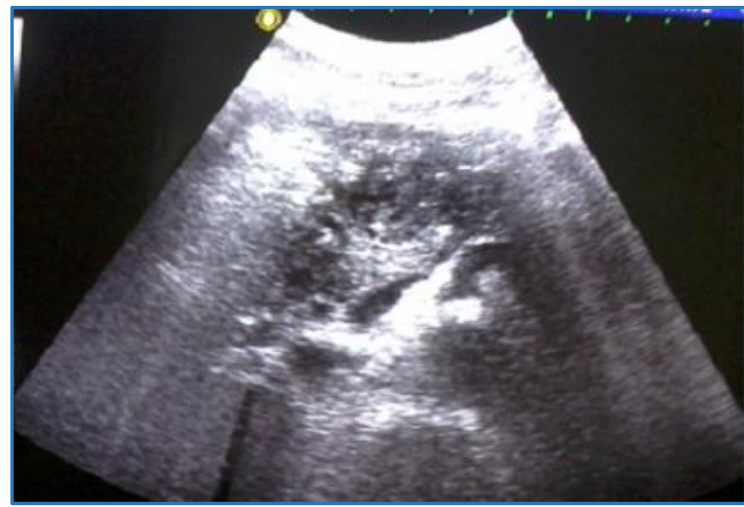

Figure $3 a$

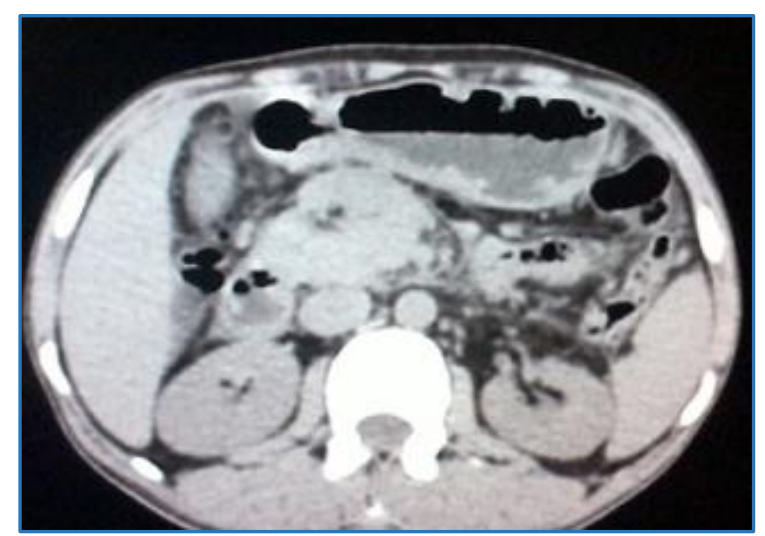

Figure $3 a$ \& b. Mass Lesion in Head of Pancreas

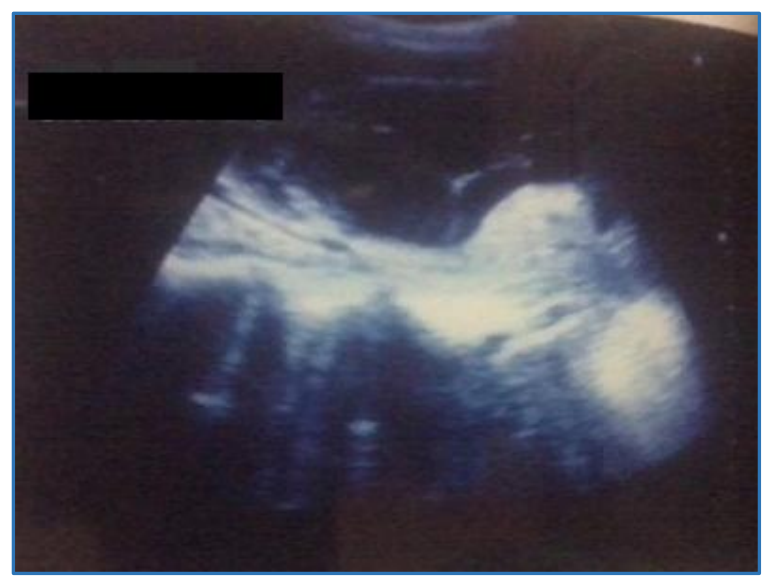

Figure 4a

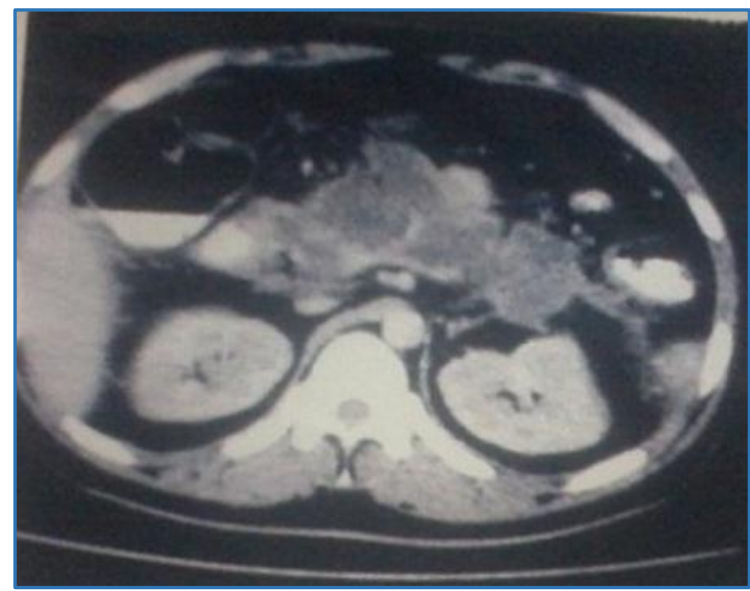

Figure 4a \& b. Cystic Mass Lesion in the Head and Body Regions of Pancreas

\section{RESULTS}

The sensitivity and specificity of contrast enhanced computed tomography in the diagnosis of pancreatic tumours is $88.37 \%$ and $71.43 \%$ respectively. The sensitivity and specificity of ultrasound in the diagnosis of pancreatic tumours is $76.10 \%$ and $62.50 \%$ respectively.

\section{DISCUSSION}

This study included 50 patients referred to our department with a high suspicion of pancreatic neoplasm. Of these 50 patients, CT detected 40 cases to be positive. Ultrasound detected only 35 of these 40 patients. Ultrasound did not detect 5 cases which were positive on CT.

\begin{tabular}{|c|c|c|}
\hline $\begin{array}{c}\text { Cases with High Suspicion } \\
\text { of Pancreatic Neoplasm } \\
\text { Based on Clinical \& } \\
\text { Laboratory Findings }\end{array}$ & $\begin{array}{c}\text { Positive } \\
\text { on } \\
\text { CT }\end{array}$ & $\begin{array}{c}\text { Positive } \\
\text { on } \\
\text { Ultrasound }\end{array}$ \\
\hline 50 & 40 & 35 \\
\hline
\end{tabular}

Out of 10 cases found negative on CT, for pancreatic neoplasms 5 cases on further evaluation were found to have pancreatic neoplasms, of which 4 cases were confirmed as ductal adenocarcinomas on further imaging and one case as small serous cystadenoma. Thus, the sensitivity of CT in detecting pancreatic neoplasms was $88.37 \%$

Out of 40 cases positive on CT, further followup showed that 38 cases were positive for pancreatic neoplasms and 2 cases were negative. Thus, the specificity of CT in detecting pancreatic neoplasms was $71.43 \%$

On ultrasound, 35 cases were found positive and 15 cases were found negative for pancreatic neoplasms. Out of 15 cases found negative on ultrasound, CT evaluation showed 5 cases to be positive for pancreatic neoplasms which was confirmed on followup. Out of these 5 cases, in 4 cases visualisation of pancreas was poor due to gas distended bowel loops. Of these 4 cases, 3 were found to be ductal adenocarcinoma on CT ( 2 in the body and 1 in the tail region) and other being serous cystadenoma in the body of pancreas. In another case with clinical features s/o pancreatic neuroendocrine tumour though the pancreas was well visualised on ultrasound, mass was missed due to its isoechoic nature to adjacent parenchyma. On CT it was detected by the evident contrast enhancement. Five other cases which were negative both on ultrasound and CT were also found positive on further imaging. Thus, the sensitivity of ultrasound was $76.10 \%$.

Out of 35 cases found positive on ultrasound, three cases were found negative on further imaging. Out of these 3 false positive cases, two were positive on CT also, but finally proved negative on MR imaging. And another case was found normal on CT and the same was confirmed on further imaging. Thus, the specificity of ultrasound was $62.50 \%$.

\begin{tabular}{|c|c|c|c|}
\hline Sl. No. & Diagnosis & No. of Cases & $\%$ \\
\hline 1. & Ductal adenocarcinoma & 31 & $77.5 \%$ \\
\hline 2. & Cystic neoplasms & 6 & $15 \%$ \\
\hline 3. & Neuroendocrine tumours & 1 & $2.5 \%$ \\
\hline 4. & Lymphomas & 1 & $2.5 \%$ \\
\hline 5. & Metastasis & 1 & $2.5 \%$ \\
\hline 6. & Normal on CT/USG & 10 & $5 \%$ \\
\hline & Total & 50 & \\
\hline \multicolumn{4}{|c|}{ Summary of Cases } \\
\hline
\end{tabular}


In the present study, out of 40 positive cases; 31 cases were diagnosed as ductal adenocarcinomas, 6 cases as cystic neoplasms, 1 case as pancreatic neuroendocrine tumour, 1 case as lymphoma, 1 case as metastasis to pancreas. 10 cases were found normal.

\begin{tabular}{|c|c|c|c|}
\hline Sl. No. & Resectability & No. of Cases & \% \\
\hline 1. & Non-resectable & 31 & $77.5 \%$ \\
\hline 2. & Resectable & 9 & $22.5 \%$ \\
\hline \multicolumn{4}{|c|}{ Resectability of Pancreatic Neoplasms } \\
\hline \multicolumn{4}{|c|}{}
\end{tabular}

In the present study, out of 40 cases, $77.5 \%$ cases were detected to be non-resectable due to local or distant spread of tumour. $22.5 \%$ of cases were detected to be resectable.

\section{CONCLUSION}

In the present study of 50 patients, an attempt was made to evaluate the efficiency of CT and ultrasound in detecting and evaluating various pancreatic neoplasms.

The imaging findings on US and CT had favourable results and had been impressive in identifying associated findings also, thus giving the clinician a broader perspective in instituting prompt management.

The use of ultrasound is non-invasive, non-ionising taking only few minutes, easy to perform and interpret, cost effective with good repeatability and reliability. In the present study, USG was less sensitive and specific in the detection of pancreatic neoplasms when compared to CT. It was not able to diagnose small isoechoic solid masses which were diagnosed on CECT by contrast enhancement. Difficulty also occurred with ultrasound in accurate assessment of local extension and small sized liver metastases. This study showed the sensitivity and specificity of ultrasound as $76.10 \%$ and $62.50 \%$ respectively.

Contrast enhanced CT has emerged as a better and superior modality in detecting various pancreatic neoplasms and it was more specific and sensitive when compared to ultrasound. CECT was able to detect the extent of adjacent organ involvement more accurately than with ultrasound. This study showed sensitivity and specificity of computed tomography as $88.37 \%$ and $71.43 \%$ respectively.

\section{REFERENCES}

[1] Sutton D. Textbook of Radiology and Medical Imaging. $7^{\text {th }}$ edn. Churchill Livingstone Vol 1, Section 3, pg 795.

[2] Hanbidge AE. Cancer of the pancreas: the best image for early detection-CT, MRI, PET or US? Can J Gastroenterol 2002;16(2):101-5.

[3] Wang Y, Miller FH, Chen ZE, et al. Diffusion-weighted MR imaging of solid and cystic lesions of the pancreas. Radiographics 2011;31(3):E47-64.

[4] Kartalis N, Lindholm TL, Aspelin P, et al. Diffusionweighted magnetic resonance imaging of pancreas tumours. Eur Radiol 2009;19(8):1981-90.

[5] Low G, Panu A, Millo N, et al. Multimodality imaging of neoplastic and nonneoplastic solid lesions of the pancreas. Radiographics 2011;31(4):993-1015. 